\title{
Pituitary Gland
}

\author{
SITE OF SHORT-LOOP FEEDBACK FOR \\ LUTEINIZING HORMONE IN THE RABBIT
}

\author{
Nilsa Patritti-Laborde, Ada R. Wolfsen, David Heber, and William D. Odell, \\ Division of Endocrinology and Metabolism, Departments of Medicine and \\ Physiology, Harbor-UCLA Medical Center, \\ Torrance, California 90509
}

A B S T R A C T The development of a species specific radioimmunoassay for rabbit luteinizing hormone $(\mathrm{LH})$ has permitted the direct demonstration of $\mathrm{LH}$ feedback control of LH secretion (short-loop feedback control). In previous studies we showed that small bolus injections of human $\mathrm{LH}(\mathrm{hLH})$ intravenously administered to castrate female rabbits suppressed rabbit $\mathrm{LH}$ for 20-30 min. Human LH had no effect on rabbit follicle-stimulating hormone secretion. This control system was responsive to amounts of hLH estimated to be present in blood of eugonadal men and women. These studies were designed to determine whether this feedback control was exerted at a pituitary or hypothalamic level. Two groups of studies were carried out: $(a)$ in vivo studies: Rabbit $\mathrm{LH}$ was quantified in the blood of castrated female New Zealand White rabbits receiving either constant $\mathrm{hLH}$ perfusion $(2.75 \mathrm{IU} / \mathrm{min})$ or saline perfusion, plus a bolus injection of $0.5,6$, or $20 \mu \mathrm{g}$ of gonadotropin-releasing hormone (GnRH). Human $\mathrm{LH}$ decreased the response to 6 and $20 \mu \mathrm{g}$ of GnRH by 31 and $36 \%$, respectively, and abolished the response to $0.5 \mu \mathrm{g}$, GnRH. (b) in vitro studies: Rabbit pituitary slices were incubated in the presence of medium alone, medium plus hLH $(25 \mathrm{mIU} / \mathrm{ml})$, medium plus $\mathrm{GnRH}$ $(20 \mu \mathrm{g} / \mathrm{ml})$, and medium plus both GnRH and hLH. hLH decreased basal rabbit LH release into the medium and abolished GnRH-stimulated rabbit $\mathrm{LH}$ release. hLH had no effect on rabbit follicle-stimulating hormone release. From these results we conclude that a direct and specific feedback control of $\mathrm{LH}$ on LH exists at a pituitary level.

This work was published in abstract form in the Program of the 61st Annual Meeting of the Endocrine Society, 13-15 June 1979 and in Clin. Res. 27: 23A. (Abstr.)

Received for publication 17 January 1979 and in revised form 1.9 June 1.97 .9 .

\section{INTRODUCTION}

Previous studies from this laboratory have directly demonstrated the existence of a short-loop feedback mechanism for control of luteinizing hormone $(\mathrm{LH})^{1}$ secretion in rabbits $(1,2)$. These studies showed that as little as $1 \mathrm{IU}$ of human $\mathrm{LH}(\mathrm{hLH})$ given as an intravenous bolus injection into ovariectomized rabbits decreased basal rabbit LH (rbLH) levels by more than $50 \%$ and indicated that this regulatory feedback is adequately sensitive to operate at physiological concentrations of LH. In addition, this is the only entirely specific control system known for LH; even large doses of LH had no influence on follicle-stimulating hormone (FSH) concentration and FSH and thyrotropin-stimulating hormone failed to change $\mathrm{LH}$ concentrations. These studies were designed to determine the site of action for this short-loop feedback mechanism. Both in vivo and in vitro experiments demonstrated that $\mathrm{LH}$ acts at the pituitary level to control its own secretion.

\section{METHODS}

In vivo studies. Adult female New Zealand White rabbits were castrated $5 \mathrm{~d}$ before the study and permanent silastic catheters were placed in both external jugular veins, extending to different levels of the right atrium. These animals remained healthy and could be studied without anesthesia over several weeks' time. Unanesthetized animals were perfused for $240 \mathrm{~min}$ with sterile saline solution $(0.19 \mathrm{ml} /$ $\min , 0.9 \% \mathrm{NaCl})$ as the control group, or $\mathrm{hLH}^{2}(2.75 \mathrm{IU} / \mathrm{min})$

\footnotetext{
${ }^{1}$ Abbreviations used in this paper: FSH, follicle-stimulating hormone; GnRH, gonadotropin-releasing hormone; hLH, human LH; LH, luteinizing hormone; rb-g, rabbit gamma globulin; rbLH, rabbit $\mathrm{LH}$.

${ }^{2}$ Highly purified hLH of immunochemical grade was kindly supplied by The Hormone Distribution Office, National Institutes of Arthritis and Metabolic Diseases and perfused in $0.9 \% \mathrm{NaCl}$.
} 
through an indwelling catheter connected to a Harvard perfusion pump (Harvard Apparatus Co., Inc., Millis, Mass.). To saturate the distribution pool rapidly, animals perfused with hLH were primed with a bolus injection of $10 \mathrm{IU}$ of $\mathrm{hLH}$. In both control groups and hLH infused groups, bolus injections of $0.5,6$, or $20 \mu \mathrm{g}$ of gonadotropin-releasing hormone (GnRH diacetate, Bachem Inc., Torrance, Calif.) were administered via the second catheter after $60 \mathrm{~min}$ of perfusion. The various doses of GnRH with or without concomitant hLH perfusion were randomly evaluated, so as not to allow bias due to order of administration. The same animals were restudied at 3- to 7-d intervals, but no animal was studied more than 4 wk after castration, since our earlier studies (1) suggested sensitivity of short-loop feedback decreases after several weeks. Blood samples were obtained in relation to treatment at the following times: $0,10,25 \mathrm{~min}$, (saline or hLH perfusion started) $30,40,50,60 \mathrm{~min},(\mathrm{GnRH}$ administered) $65,75,90,120,150,180$, and $240 \mathrm{~min}$. For estimating GnRH response, the mean of the 30-, 40-, 50-, and 60-minvalues was used as basal rbLH.

In vitro studies. Two independent experiments were performed with the following design: For each experiment, 20 adult female New Zealand White rabbits were sacrificed $5 \mathrm{~d}$ after castration and pituitaries were collected on ice and manually sliced with a scalpel into sections $\cong 1 \mathrm{~mm}$ thick. The slices were placed in 25-ml Erlenmeyer flasks containing $2 \mathrm{ml}$ of medium 199 (Grand Island Biological Co., Grand Island, N. Y.). All flasks were preincubated for $30 \mathrm{~min}$ at $37^{\circ} \mathrm{C}$ in a Dubnoff incubator, in the presence of $95 \% \mathrm{O}_{2}$ and $5 \% \mathrm{CO}_{2}$ atmosphere. Media were then changed, and four groups of five flasks each were incubated separately with various hormone combinations: (a) $2 \mathrm{ml}$ of medium alone (control); (b) $2 \mathrm{ml}$ of medium with $\mathrm{GnRH} 20 \mathrm{ng} / \mathrm{ml}$; (c) $2 \mathrm{ml}$ of medium with $25 \mathrm{IU} / \mathrm{ml}$ of hLH; (d) $2 \mathrm{ml}$ of medium with both $\mathrm{GnRH}$ and $\mathrm{hLH}$ in the same concentrations given above. Medium was removed from each flask for rbLH determination and replaced with $2 \mathrm{ml}$ of the corresponding fresh medium at 30,60 , and $120 \mathrm{~min}$ of incubation time. In further studies for specificity, pituitary slices were incubated with (a) medium alone; (b) GnRH $(20 \mathrm{ng} / \mathrm{ml})$; (c) human FSH $(2.5$ $\mathrm{ng} / \mathrm{ml}$ ) (Dr. A. F. Parlow preparation, Harbor-UCLA Medical Center); (d) rabbit gamma globulins (rb-g globulin, $5 \mathrm{ng} / \mathrm{ml}$ ) (fraction II, Pentex Miles Laboratories, Inc., Elkhart, Ind.); (e) combination of $\mathrm{GnRH}$ plus $\mathrm{FSH}$; and $(f) \mathrm{GnRH}$ plus rb-g globulin. All media were kept frozen at $-70^{\circ} \mathrm{C}$ until assayed for rbLH levels. A specific radioimmunoassay method was used, as described (2), which does not cross react with hLH. Antibody and standard rbLH were kindly supplied by Dr. A. Parlow and [ $\left.{ }^{125} \mathrm{I}\right] \mathrm{rbLH}$ was prepared by the chloramine T method and purified by a concanavalin A Sepharose (Pharmacia Fine Chemicals, Piscataway, N. J.) column as described in a previous paper (3).

\section{RESULTS}

In vivo studies. Human $\mathrm{LH}$ administered as a constant perfusion decreased the response to bolus injections of GnRH. Fig. 1 shows the response of a single rabbit studied on two occasions $3 \mathrm{~d}$ apart. $20 \mu \mathrm{g}$ of GnRH produced a rapid increase in rbLH during saline perfusion. When the same animal was given $20 \mu \mathrm{g}$ of GnRH during hLH perfusion, the response to GnRH was decreased $41 \%$.

Fig. 2 shows the mean response $( \pm \mathrm{SEM})$ of all animals studied. The response to 6 and $20 \mu \mathrm{g}$ of $\mathrm{GnRH}$ was

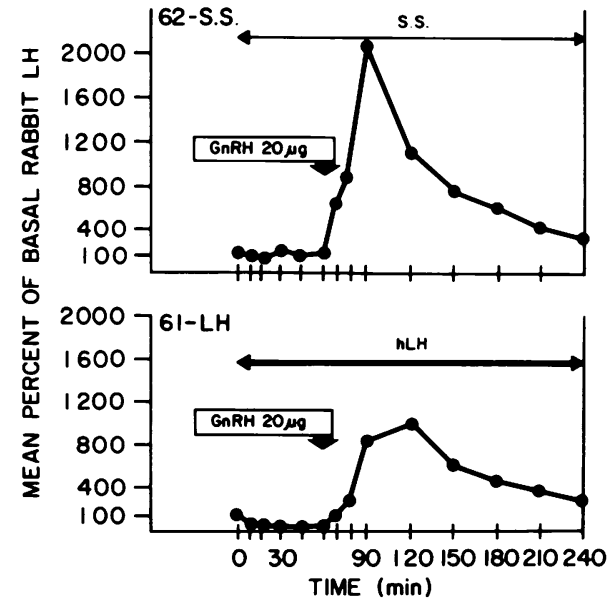

FIgURE 1 In vivo study. Pattern of response in a single animal of rLH after a bolus injection of $20 \mu \mathrm{g}$ of $\mathrm{GnRH}$, during saline solution (S.S.) (upper panel), or during hLH perfusion lower panel. The percentage of change in $\mathrm{rbLH}$ is plotted on the $y$-axis. The $100 \%$ value is the average of control values obtained at 15, 5 , and 0 min before the perfusion period. Arrows indicate the time of the intravenous bolus injection of $\mathrm{GnRH}$. The two studies were performed in the same animal on different days.

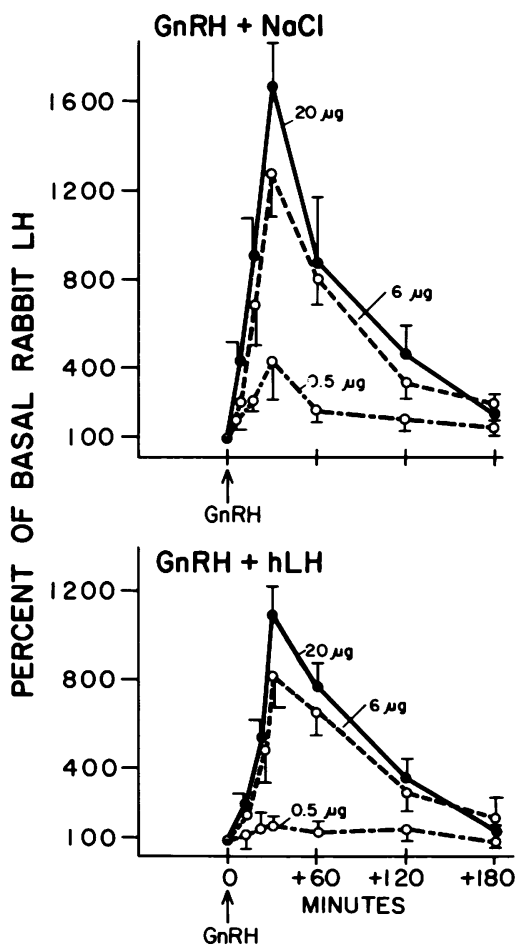

Figure 2 In vivo studies. Average effects of $0.5,6$, and 20 $\mu \mathrm{g}$ of GnRH on rLH during saline solution (S.S.) infusion ( $\mathrm{NaCl}$ ) (upper panel) or hLH $2.75 \mathrm{IU}$ (lower panel) perfusion. Results are expressed as percent increase of $\mathrm{rLH}$ in relation to basal values. Brackets enclose $1 \mathrm{SEM}$. hLH decreased the response to all doses of $\mathrm{GnRH}(P=₹ 0.05)$. 


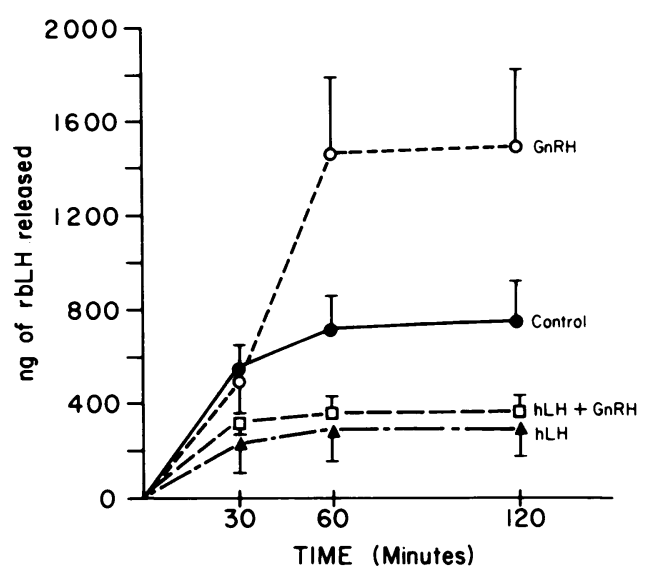

Figure 3 In vitro studies. $\mathrm{rLH}$ released (calculated as total LH in medium at time indicated) into the medium when pituitaries were incubated in the presence of medium alone (control), $50 \mathrm{IU}$ of hLH, $40 \mathrm{ng}$ of $\mathrm{GnRH}$ and the combination of both hLH and GnRH in the concentrations given. Five pituitaries were incubated in each group, results represent mean \pm SEM. hLH inhibited both basal LH release and GnRH stimulated $(P<0.05)$.

decreased by 31 and $36 \%$, respectively by hLH perfusion ( $P=₹ 0.05$ for each dose) and the response to $0.5 \mu \mathrm{g}$ was abolished. Assay of blood human LH concentrations after 30 and $60 \mathrm{~min}$ of perfusion revealed concentrations of 46 and $52 \mathrm{mIU} / \mathrm{ml},{ }^{3}$ respectively.

In vitro studies. Human LH inhibited basal release into the medium as well as GnRH-stimulated LH release at all time periods studied. Fig. 3 demonstrates these data. Human LH had no influence on rbFSH release. Similarly, incubation of pituitary slices with rabbit gamma globulin or human FSH produced no effects on rbLH released into the medium (Fig. 4).

\section{DISCUSSION}

We have previously reported several interesting characteristics of the short-loop feedback mechanism of control of $\mathrm{LH}$ secretion in rabbits. This system is remarkably sensitive and also specific $(1,2)$. Both characteristics indicate that it may have an important physiological role in regulation of $\mathrm{LH}$. In previous studies Corbin (4), David et al. (5), Mc Cann et al. (6), and Ojeda and Ramirez (7) suggested the hypothalamus was the site for this control mechanism. However, considering the very complex circulatory system connecting hypothalamus and pituitary, alternative interpretations may be possible (8). In this study, we directed our attention to the anatomic site for short-loop feedback control.

\footnotetext{
${ }^{3}$ In terms of second international reference preparation of human menopausal gonadotropin (IRP-HMG No. 2).
}

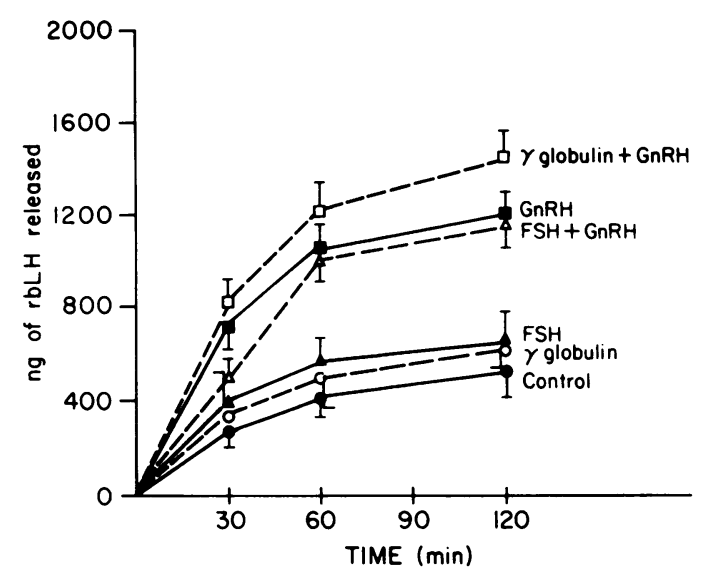

Figure 4 In vitro studies. rLH released into the medium (sampled at 30,60 , and $120 \mathrm{~min}$ ) when pituitaries were incubated with medium alone (control), $5 \mathrm{ng}$ of rb-g, $3.5 \mathrm{ng}$ human FSH and the combination of GnRH plus FSH and GnRH plus rb-g. Brackets enclose 1 SE.M. Human FSH and rb-g produced no effects on LH release.

As we designed these studies, we first took the viewpoint that the hypothalamus was the exclusive site of feedback. If this were true, we should not see any effects of hLH on rbLH release in the in vitro incubation studies. In addition, if the hypothalamus were the exclusive site of control, one might expect GnRH stimulation of rbLH secretion in vivo to be unaffected by infusion of hLH. Surprisingly, in both in vitro and in vivo studies, acute increases in medium or blood LH concentrations respectively inhibited basal and $\mathrm{GnRH}$ stimulated $\mathrm{rbLH}$ secretion. These data therefore indicate that the short-loop feedback control of $\mathrm{LH}$ operates directly at a pituitary level. This effect was specific in that there was no inhibition of $\mathrm{GnRH}$ stimulated rbLH by human FSH or rabbit gamma globulin. It is possible that an additional short-loop control mechanism also exists at a hypothalamic level, but such an additional level of control is not necessary to explain the data presented herein.

We conclude that an LH feedback control system on LH secretion exists at the pituitary gland.

\section{ACKNOWLEDGMENTS}

We express our gratitude to Dr. A. Parlow and the National Institute of Arthritis, Metabolism and Digestive Diseases Hormone Distribution Program, Department of Obstetrics \& Gynecology, Harbor-UCLA Medical Center for supplying the reagents for rbLH radioimmunoassay and for the immunochemical grade human LH used in these studies.

\section{REFERENCES}

1. Molitch, M., M. Edmonds, E. Jones, and W. D. Odell, 1976. Short-loop feedback control of luteinizing hormone (LH) in the rabbit. Am. J. Physiol. 230: 907-910. 
2. Patritti-Laborde, N., and W. D. Odell. 1978. Short-loop feedback of LH. Dose response relations and specificity. Fertil. Steril. 30: 456-460.

3. Patritti-Laborde, N., Y. Yoshimoto, A. R. Wolfsen, and W. D. Odell. 1979. Improved method of purifying some radiolabelled glycopeptide hormones. Clin. Chem. 25: 163-165.

4. Corbin, A. 1966. Pituitary and plasma LH of ovariectomized rats with median eminence implants of LH. Endocrinology. 78: 893-896.

5. David, M. A., F. Fraschini, and L. Martini. 1966. Control of LH secretions: role of a short feedback mechanism. Endocrinology. 78: 893-896.

6. Mc Cann, S. M., A. P. S. Dhariwal, and J. C. Porter. 1968. Regulation of adenohypophysis. Annu. Rev. Physiol. 30: $589-640$.

7. Ojeda, S. R., and V. D. Ramirez. 1969. Automatic control of LH and FSH secretion by short feedback circuits in immature rats. Endocrinology. 84: 786-797.

8. Bergland, R. M., and R. B. Page. 1979. Pituitary-brain vascular relations: a new paradigm. Science (Wash. D. C.). 204: 18-24. 